\title{
Target Prediction of Xinyi San for Rhinitis Based on Network Pharmacology
}

\author{
Lingdi Wang*, Ying Wang, Yingxue Lin, Xin Meng \\ Department of Pharmacy, The Affiliated Hospital of Chengde Medical College, Chengde, China \\ Email: *363121249@qq.com
}

How to cite this paper: Wang, L.D., Wang, Y., Lin, Y.X. and Meng, X. (2022) Target Prediction of Xinyi San for Rhinitis Based on Network Pharmacology. Chinese Medicine, 13, 1-14.

https://doi.org/10.4236/cm.2022.131001

Received: December 27, 2021

Accepted: February 27, 2022

Published: March 2, 2022

Copyright $\odot 2022$ by author(s) and Scientific Research Publishing Inc. This work is licensed under the Creative Commons Attribution International License (CC BY 4.0).

http://creativecommons.org/licenses/by/4.0/ (c) (i) Open Access

\begin{abstract}
Objective: To analyze the potential mechanism of Xinyi San in treating rhinitis through network pharmacology. Methods: In the database of Traditional Chinese Medicine Systems Pharmacology (TCMSP), chemical composition and potential targets of Xinyi San were got, and the target genes of rhinitis of Xinyi San were extracted from GeneCards databases. Then we constructed protein-protein interactions (PPI) network of target genes, and then analyzed the Key genes in GO analysis and KEGG analysis. Results: We got 97 components, 53 potential therapeutic targets, 1009 GO items and 92 pathways in our study. The main pathways included Lipid and atherosclerosis, Chemical carcinogenesis-receptor activation, PI3K-Akt signaling pathway, Human cytomegalovirus infection, Prostate cancer, etc. Conclusion: Xinyi San plays a role in treating rhinitis through multiple components, multiple targets and multiple pathways.
\end{abstract}

\section{Keywords}

Rhinitis, Network Pharmacology, Xinyi San

\section{Introduction}

Rhinitis is the inflammation of nasal mucosa caused by viruses, bacteria, allergens, various physical and chemical factors and some systemic diseases, and which with nasal congestion, runny nose, nasal pruritus, sneezing, decreased smell, headache and dizziness as the main clinical manifestations. Nowadays, rhinitis has become a global health problem. In China, the incidence rate of rhinitis is as high as $37 \%$, and the number of rhinitis cases is increasing at a rate of 20 million - 3 per million per year [1]. Modern medical treatment of rhinitis is mainly to control and alleviate symptoms. However, the side effects of therapeutic drugs are obvious, and it is easy to relapse. Traditional Chinese medicine has 
irreplaceable advantages in the treatment of rhinitis. It has significant curative effect and minor side effects.

Xinyi San originated from Yan's Jisheng recipe written by Yan Yonghe in the Song Dynasty [2]. The whole recipe was composed of 10 drugs such as xinyi (Magnoliae Flos), xixin (Asari Radix Et Rhizoma), gaoben (Ligustici Rhizoma Et Radix), shengma (Cimicifugae Rhizoma), chuanxiong (Chuanxiong Rhizoma), mutong (Akebiae Caulis), fangfeng (Saposhnikoviae Radix), qianghuo (Notopterygii Rhizoma Et Radix), gancao (Glycyrrhizae Radix Et Rhizoma) and baizhi (Angelicae Dahuricae Radix). It is effective to evacuate wind cold, dredge nose orifices, and mainly treats lung deficiency, wind cold, damp and heat. In addition, the nose is blocked, the nose is full of tears, or the breath is blocked, or there is no smell. Modern clinical research also shows that Xinyi San has a good therapeutic effect on rhinitis [3] [4]. However, due to the complex clinicopathology of rhinitis and more chemical components contained in Xinyi San, the mechanism of its treatment of rhinitis at the molecular level is not clear. Network pharmacology is a branch of pharmacology that uses network methods to analyze the "multi-component, multi-target and multi-channel" synergistic relationship between drugs, diseases and targets. It can build a complex network between drugs, components, targets and diseases to explore the action mechanism of drugs [5]. Therefore, based on network pharmacology, this study explores the pharmacodynamic material basis and potential mechanism of Xinyi San in the treatment of rhinitis, so as to provide reference for further basic research, clinical application and new drug research and development.

\section{Materials and Methods}

\subsection{Screened for Components and Targets of Xinyi San}

Based on TCMSP (http://tcmspw.com), the candidate chemical components and corresponding protein targets were searched with xinyi, xixin, gaoben, shengma, chuanxiong, mutong, fangfeng, qianghuo, gancao and baizhi as keywords. And with UniProt (https://www.uniprot.org/) query the gene name corresponding to the target protein for standardization.

\subsection{Screened for Rhinitis Targets}

Entered the keyword "rhinitis" in the Genecards database

(https://www.genecards.org) to search for disease targets which were related to rhinitis. We integrated targets and eliminated duplicate targets. Then, we obtained the total targets.

\subsection{Venn Analysis of Potential Targets in the Treatment of Rhinitis}

The potential active ingredient and targets of the Xinyi San and the rhinitis were mapped the intersection through the Venny platform (version 2.1,

http://bioinfogp.cnb.csic.es/tools/venny/). And then we obtained the potential targets of Xinyi San in the treatment of rhinitis. 


\subsection{Constructed Disease - Single Drug - Active Ingredient - Target" Network}

According to the above screening results, Cytoscape 3.7.2 software constructed the relationship network model of "disease-single drug-active component-target". The node represents single drug, active component and action target, and the edge represents the relationship between single drug-active component-target.

\subsection{Constructed PPI Network}

String database (https://string-db.org) is a database for searching the interaction between proteins. We imported the intersection targets of Xinyi San obtained by Venn analysis into the string database, with the species' limition as "Homo sapiens", and then we removed the isolated targets. The screening criteria were confidence (0.4).

\subsection{Gene Enrichment Analysis}

In order to analyze the biological function and biological signal pathway of Xinyi San on rhinitis, GO functional enrichment analysis and KEGG pathway enrichment analysis were performed on R4 1.1 software. With $\mathrm{p}<0.05$, the top 20 biological processes and pathways were selected according to the number of targets involved in each entry and $\lg \mathrm{P}$ value.

\section{Results}

\subsection{Screened Components of Xinyi San}

A total of 97 potential active components of Xinyi San were finally screened by TCMSP platform, the correspondent target proteins of each active ingredient were also obtained (see Table 1).

Table 1. The basic information of potential active components in Xinyi San.

\begin{tabular}{cccccc}
\hline Source & Mol ID & Molecule Name & MW & OB (\%) & DL \\
\hline xixin & MOL000018 & $(+/$-)-Isoborneol & 154.28 & 86.98413 & 0.05275 \\
xixin & MOL000715 & l-Menthone & 154.28 & 57.89753 & 0.029429 \\
xixin & MOL000254 & eugenol & 164.22 & 56.2419 & 0.036518 \\
xixin & MOL001972 & Pulegone & 152.26 & 51.59647 & 0.029676 \\
xixin & MOL000198 & (R)-linalool & 154.28 & 39.8043 & 0.022686 \\
xixin & MOL000122 & 1,8-cineole & 154.28 & 39.72922 & 0.049041 \\
xixin & MOL000169 & alpha-Guaiene & 204.39 & 25.93463 & 0.072089 \\
xixin & MOL000069 & palmitic acid & 256.48 & 19.29656 & 0.098573 \\
xixin & MOL003127 & Germacrene D & 204.39 & 19.22251 & 0.057074 \\
xixin & MOL000860 & stearic acid & 284.54 & 17.82543 & 0.14086 \\
xinyi & MOL000018 & (+/-)-Isoborneol & 154.28 & 86.98413 & 0.05275 \\
\hline
\end{tabular}


Continued

\begin{tabular}{|c|c|c|c|c|c|}
\hline xinyi & MOL000676 & DBP & 278.38 & 64.54164 & 0.13409 \\
\hline xinyi & MOL013062 & BZQ & 182.23 & 58.62326 & 0.059888 \\
\hline xinyi & MOL000254 & eugenol & 164.22 & 56.2419 & 0.036518 \\
\hline xinyi & MOL002002 & cis-Carveol & 152.26 & 45.61104 & 0.029604 \\
\hline xinyi & MOL000122 & 1, 8-cineole & 154.28 & 39.72922 & 0.049041 \\
\hline xinyi & MOL000035 & beta-Selinene & 204.39 & 24.38821 & 0.081081 \\
\hline xinyi & MOL001393 & myristic acid & 228.42 & 21.18117 & 0.066784 \\
\hline xinyi & MOL000069 & palmitic acid & 256.48 & 19.29656 & 0.098573 \\
\hline shengma & MOL000715 & 1-Menthone & 154.28 & 57.89753 & 0.029429 \\
\hline shengma & MOL000141 & hydroxytyrosol & 154.18 & 57.56917 & 0.032051 \\
\hline shengma & MOL000254 & eugenol & 164.22 & 56.2419 & 0.036518 \\
\hline shengma & MOL001972 & Pulegone & 152.26 & 51.59647 & 0.029676 \\
\hline shengma & MOL000449 & Stigmasterol & 412.77 & 43.82985 & 0.75665 \\
\hline shengma & MOL000131 & EIC & 280.5 & 41.90444 & 0.14347 \\
\hline shengma & MOL000198 & (R)-linalool & 154.28 & 39.8043 & 0.022686 \\
\hline shengma & MOL000360 & FER & 194.2 & 39.55852 & 0.058069 \\
\hline shengma & MOL000359 & sitosterol & 414.79 & 36.91391 & 0.7512 \\
\hline shengma & MOL001393 & myristic acid & 228.42 & 21.18117 & 0.066784 \\
\hline shengma & MOL000357 & Sitogluside & 576.95 & 20.63194 & 0.6241 \\
\hline shengma & MOL000069 & palmitic acid & 256.48 & 19.29656 & 0.098573 \\
\hline shengma & MOL000879 & methyl palmitate & 270.51 & 18.08756 & 0.11594 \\
\hline qianghuo & MOL000771 & p-coumaric acid & 164.17 & 43.29024 & 0.039118 \\
\hline qianghuo & MOL000360 & FER & 194.2 & 39.55852 & 0.058069 \\
\hline qianghuo & MOL000358 & beta-sitosterol & 414.79 & 36.91391 & 0.75123 \\
\hline qianghuo & MOL000359 & sitosterol & 414.79 & 36.91391 & 0.7512 \\
\hline qianghuo & MOL000114 & vanillic acid & 168.16 & 35.47235 & 0.040917 \\
\hline qianghuo & MOL000035 & beta-Selinene & 204.39 & 24.38821 & 0.081081 \\
\hline qianghuo & MOL001393 & myristic acid & 228.42 & 21.18117 & 0.066784 \\
\hline qianghuo & MOL000357 & Sitogluside & 576.95 & 20.63194 & 0.6241 \\
\hline qianghuo & MOL000069 & palmitic acid & 256.48 & 19.29656 & 0.098573 \\
\hline qianghuo & MOL000879 & methyl palmitate & 270.51 & 18.08756 & 0.11594 \\
\hline qianghuo & MOL000860 & stearic acid & 284.54 & 17.82543 & 0.14086 \\
\hline mutong & MOL000449 & Stigmasterol & 412.77 & 43.82985 & 0.75665 \\
\hline mutong & MOL000360 & FER & 194.2 & 39.55852 & 0.058069 \\
\hline mutong & MOL000358 & beta-sitosterol & 414.79 & 36.91391 & 0.75123 \\
\hline
\end{tabular}




\section{Continued}

\begin{tabular}{|c|c|c|c|c|c|}
\hline mutong & MOL000114 & vanillic acid & 168.16 & 35.47235 & 0.040917 \\
\hline mutong & MOL000860 & stearic acid & 284.54 & 17.82543 & 0.14086 \\
\hline gaoben & MOL000612 & (-)-alpha-cedrene & 204.39 & 55.56099 & 0.10498 \\
\hline gaoben & MOL000131 & EIC & 280.5 & 41.90444 & 0.14347 \\
\hline gaoben & MOL000360 & FER & 194.2 & 39.55852 & 0.058069 \\
\hline gaoben & MOL000359 & sitosterol & 414.79 & 36.91391 & 0.7512 \\
\hline gancao & MOL000676 & DBP & 278.38 & 64.54164 & 0.13409 \\
\hline gancao & MOL000098 & quercetin & 302.25 & 46.43335 & 0.27525 \\
\hline gancao & MOL000359 & sitosterol & 414.79 & 36.91391 & 0.7512 \\
\hline fangfeng & MOL000018 & $(+/-)$-Isoborneol & 154.28 & 86.98413 & 0.05275 \\
\hline fangfeng & MOL000676 & DBP & 278.38 & 64.54164 & 0.13409 \\
\hline fangfeng & MOL001889 & Methyl linolelaidate & 294.53 & 41.93436 & 0.16791 \\
\hline fangfeng & MOL000131 & EIC & 280.5 & 41.90444 & 0.14347 \\
\hline fangfeng & MOL000198 & (R)-linalool & 154.28 & 39.8043 & 0.022686 \\
\hline fangfeng & MOL000122 & 1,8-cineole & 154.28 & 39.72922 & 0.049041 \\
\hline fangfeng & MOL000358 & beta-sitosterol & 414.79 & 36.91391 & 0.75123 \\
\hline fangfeng & MOL000359 & sitosterol & 414.79 & 36.91391 & 0.7512 \\
\hline fangfeng & MOL000114 & vanillic acid & 168.16 & 35.47235 & 0.040917 \\
\hline fangfeng & MOL000173 & wogonin & 284.28 & 30.68457 & 0.22942 \\
\hline fangfeng & MOL000035 & beta-Selinene & 204.39 & 24.38821 & 0.081081 \\
\hline fangfeng & MOL001393 & myristic acid & 228.42 & 21.18117 & 0.066784 \\
\hline fangfeng & MOL000357 & Sitogluside & 576.95 & 20.63194 & 0.6241 \\
\hline fangfeng & MOL000069 & palmitic acid & 256.48 & 19.29656 & 0.098573 \\
\hline fangfeng & MOL000879 & methyl palmitate & 270.51 & 18.08756 & 0.11594 \\
\hline fangfeng & MOL000860 & stearic acid & 284.54 & 17.82543 & 0.14086 \\
\hline fangfeng & MOL005402 & Methyl margarate & 284.54 & 17.40885 & 0.13808 \\
\hline chuanxiong & MOL000131 & EIC & 280.5 & 41.90444 & 0.14347 \\
\hline chuanxiong & MOL000198 & (R)-linalool & 154.28 & 39.8043 & 0.022686 \\
\hline chuanxiong & MOL000122 & 1, 8-cineole & 154.28 & 39.72922 & 0.049041 \\
\hline chuanxiong & MOL000359 & sitosterol & 414.79 & 36.91391 & 0.7512 \\
\hline chuanxiong & MOL000114 & vanillic acid & 168.16 & 35.47235 & 0.040917 \\
\hline chuanxiong & MOL000035 & beta-Selinene & 204.39 & 24.38821 & 0.081081 \\
\hline chuanxiong & MOL000357 & Sitogluside & 576.95 & 20.63194 & 0.6241 \\
\hline chuanxiong & MOL000069 & palmitic acid & 256.48 & 19.29656 & 0.098573 \\
\hline chuanxiong & MOL003127 & Germacrene D & 204.39 & 19.22251 & 0.057074 \\
\hline
\end{tabular}




\begin{tabular}{|c|c|c|c|c|c|}
\hline \multicolumn{6}{|l|}{ Continued } \\
\hline chuanxiong & MOL001729 & Crysophanol & 254.25 & 18.63889 & 0.2094 \\
\hline chuanxiong & MOL000879 & methyl palmitate & 270.51 & 18.08756 & 0.11594 \\
\hline chuanxiong & MOL000860 & stearic acid & 284.54 & 17.82543 & 0.14086 \\
\hline baizhi & MOL000676 & DBP & 278.38 & 64.54164 & 0.13409 \\
\hline baizhi & MOL000254 & eugenol & 164.22 & 56.2419 & 0.036518 \\
\hline baizhi & MOL000449 & Stigmasterol & 412.77 & 43.82985 & 0.75665 \\
\hline baizhi & MOL000131 & EIC & 280.5 & 41.90444 & 0.14347 \\
\hline baizhi & MOL000198 & (R)-linalool & 154.28 & 39.8043 & 0.022686 \\
\hline baizhi & MOL000360 & FER & 194.2 & 39.55852 & 0.058069 \\
\hline baizhi & MOL000358 & beta-sitosterol & 414.79 & 36.91391 & 0.75123 \\
\hline baizhi & MOL000035 & beta-Selinene & 204.39 & 24.38821 & 0.081081 \\
\hline baizhi & MOL001393 & myristic acid & 228.42 & 21.18117 & 0.066784 \\
\hline baizhi & MOL000357 & Sitogluside & 576.95 & 20.63194 & 0.6241 \\
\hline baizhi & MOL000069 & palmitic acid & 256.48 & 19.29656 & 0.098573 \\
\hline baizhi & MOL000879 & methyl palmitate & 270.51 & 18.08756 & 0.11594 \\
\hline baizhi & MOL000860 & stearic acid & 284.54 & 17.82543 & 0.14086 \\
\hline
\end{tabular}

\subsection{Venn Analysis of Targets of Xinyi San in the Treatment of Rhinitis}

The targets were predicted by Pharmmapper, and 113 potential active ingredient targets were obtained after weight removal. The obtained target names were input into UniProt database and converted into corresponding gene names. 2562 disease targets were obtained by searching "rhinitis" in genecards database. Through Venn analysis, the intersection of mapping is 53 targets that may be related to the effect of Xinyi San on rhinitis (see Figure 1).

\subsection{Constructed of Disease - Single Drug - Active Ingredient - Target" Network}

The information of disease, single drug, active ingredient and target were imported into the software Cytoscape for visualization, and the network diagram of "disease - single drug - active ingredient - target" is constructed, including 105 nodes ( 1 disease node, 10 drug nodes, 41 active ingredient nodes and 53 action target nodes) and 335 edges (see Figure 2).

\subsection{Constructed PPI Network}

53 intersecting targets obtained from Venn analysis were imported into the STRING database to build the PPI network (see Figure 3). The network contained 50 nodes (DCAF5, PCYY1A and ADRA1A were isolated targets and didn't participate in the analysis) and 369 edges. The top 30 targets were processed with 


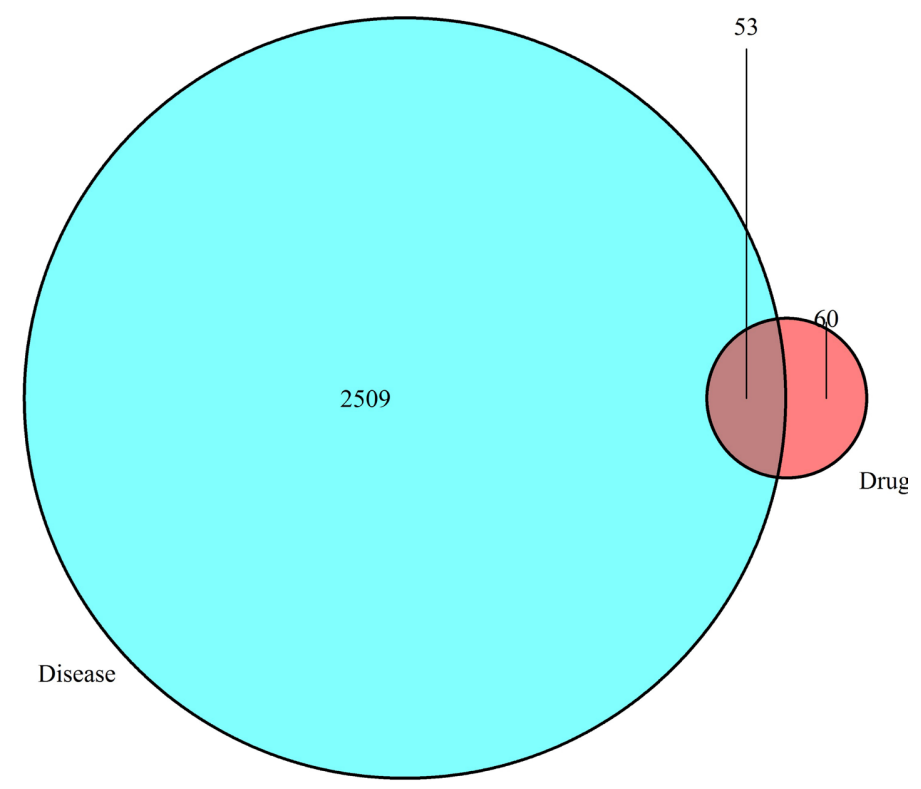

Figure 1. Venn diagram of intersection targets of Xinyi San and rhinitis.

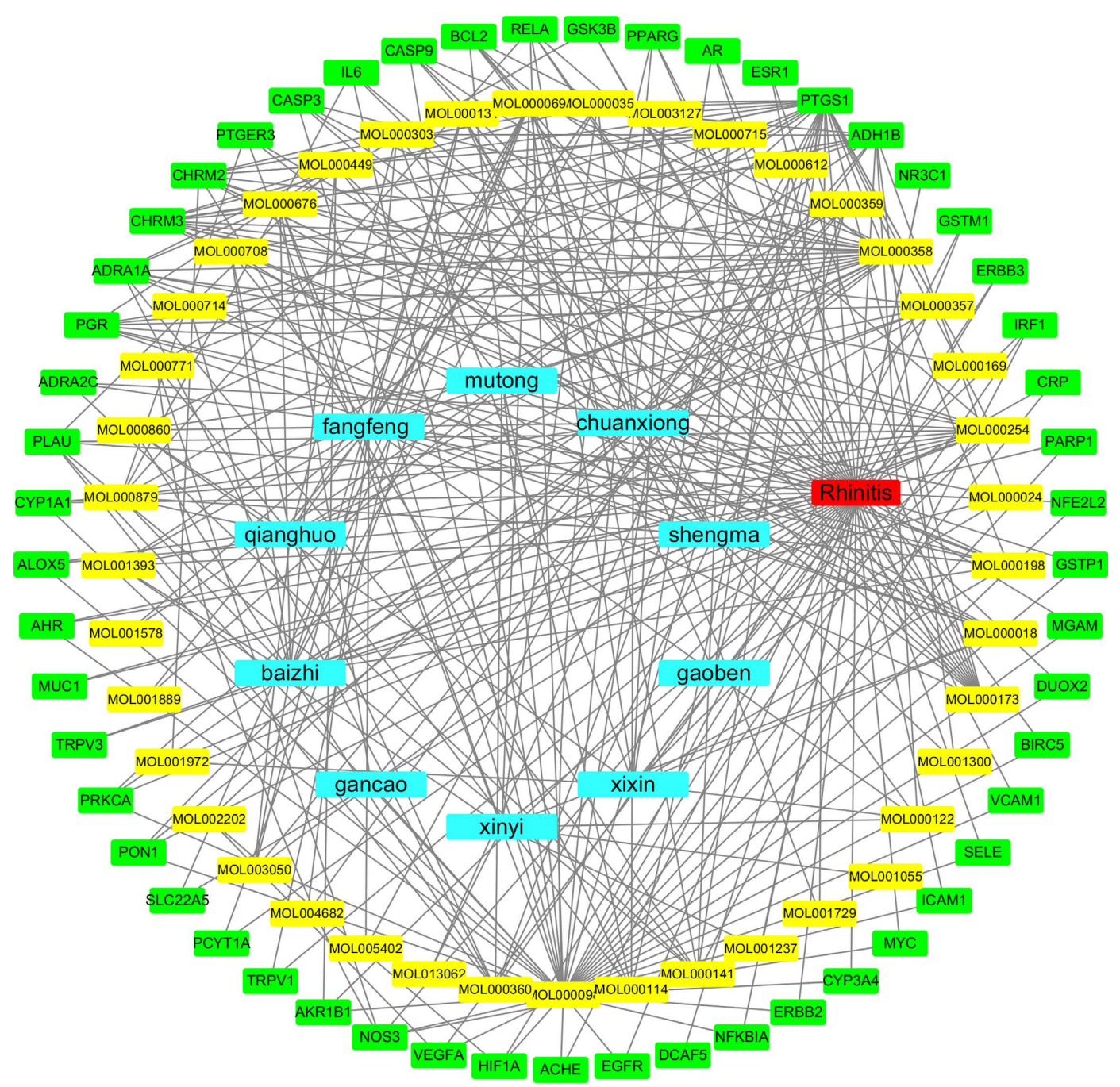

Figure 2. Composition target network of Xinyi San. 


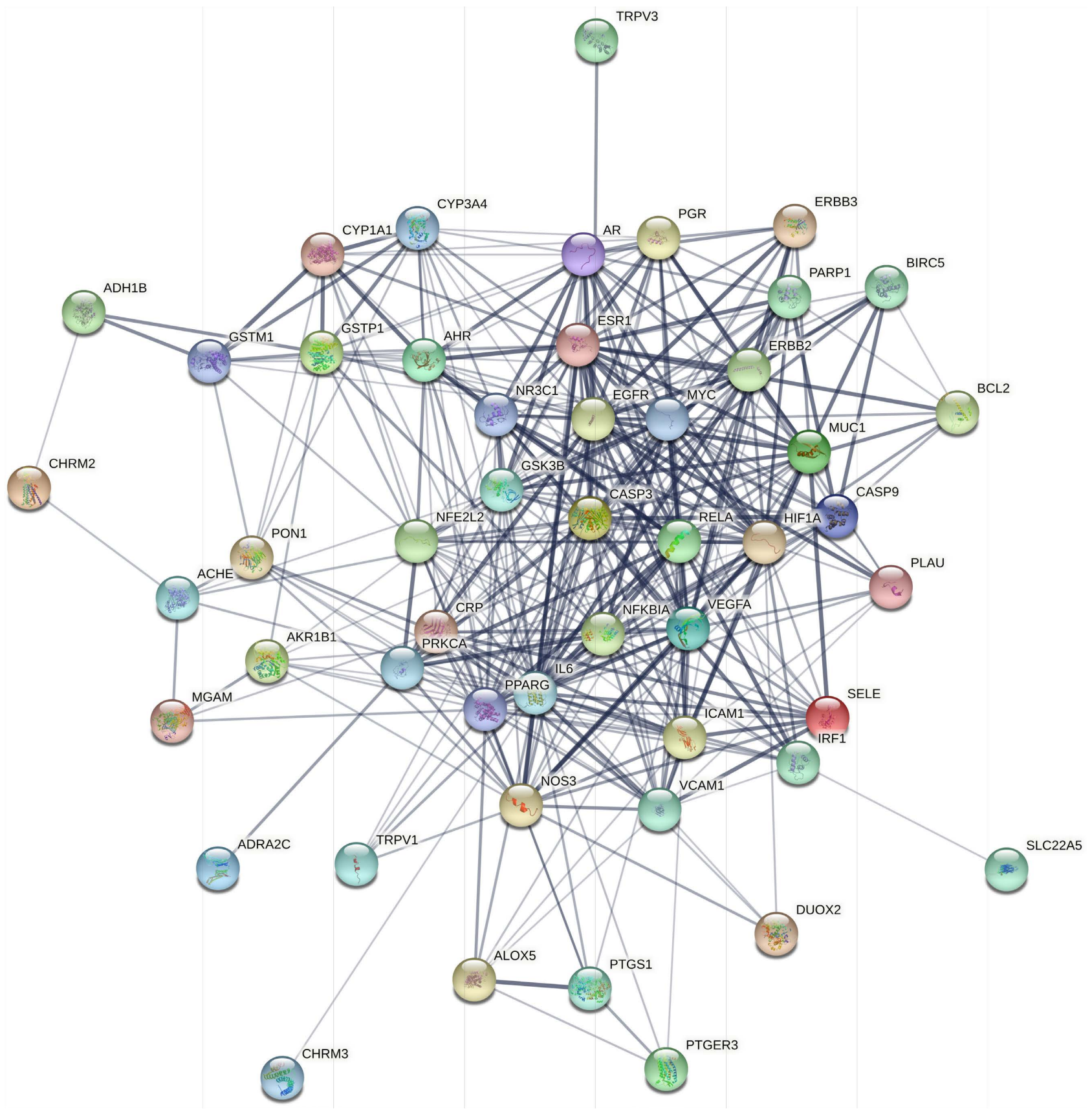

Figure 3. PPI network diagram of intersection target of Xinyi San.

$\mathrm{R}$ language to obtain the core target map (see Figure 4). The top 10 targets with visibility value were IL6, EGFR, VEGFA, HIF1A, CASP3, ESR1, PPARG, MYC, RELA, NFKBIA.

\subsection{GO Enrichment Analysis and KEGG Pathway Analysis}

Through the analysis of 53 potential targets by R software, 1009 GO entries were obtained, including 84 molecular function (MF), 8 cellular component (CC) and 917 biological process (BP) (see Figure 5). We got 92 KEGG pathways $(\mathrm{P}<0.05)$ (see Figure 6), such as Lipid and atherosclerosis, Chemical carcinogenesis 


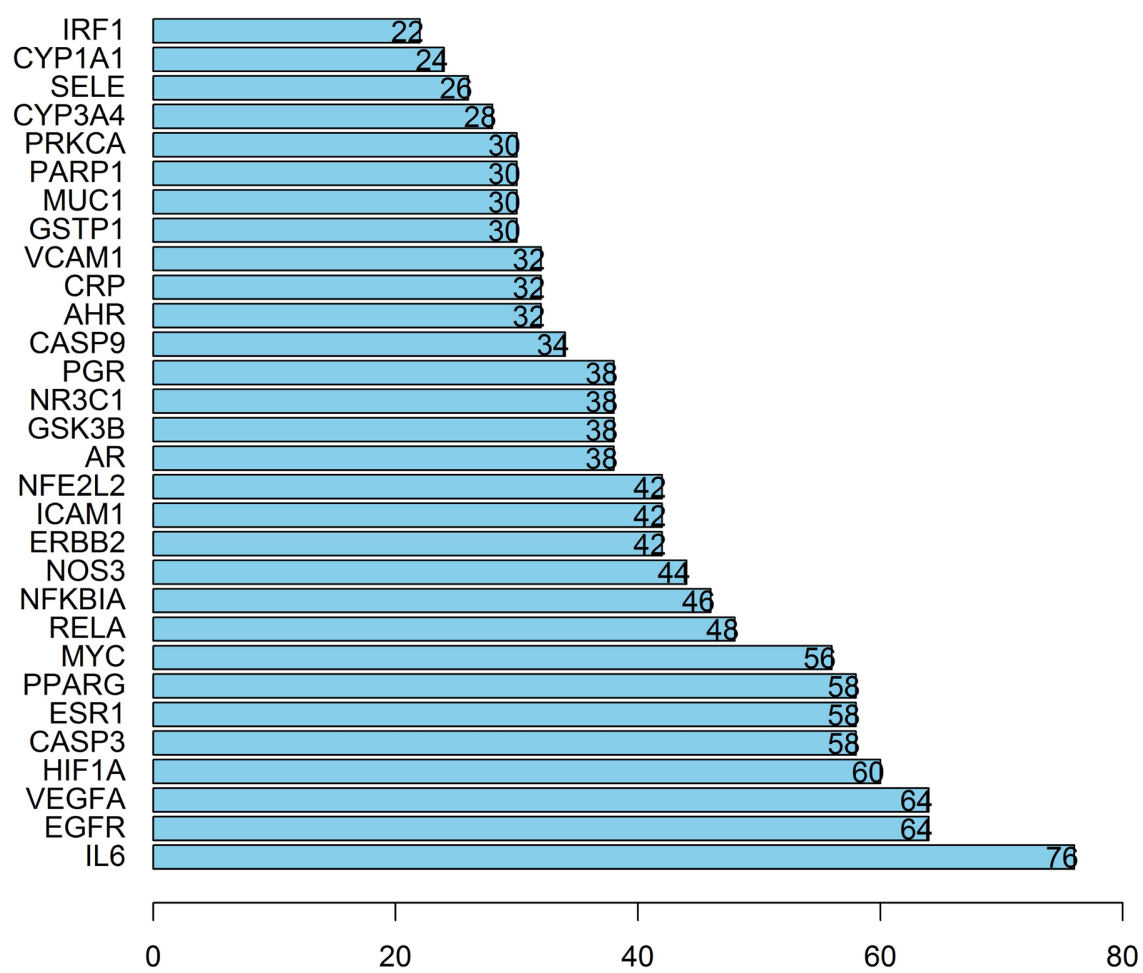

Figure 4. Key targets of Xinyi San.

receptor activation, PI3K-Akt signaling pathway, Human cytomegalovirus infection, Prostate cancer, AGE-RAGE signaling pathway in diabetic complications, Fluid shear stress and atherosclerosis, Kaposi sarcoma-associated herpesvirus infection and Calcium signaling pathway.

\section{Discussion}

Rhinitis is a high risk factor for sinusitis, nasal polyps, otitis media and bronchial asthma. This disease has a serious impact on the patient's mental state, work and life. Modern medical treatment of this disease mainly includes drug treatment, immunotherapy and surgical treatment. It mainly focuses on controlling and alleviating symptoms, but the condition is very easy to repeat.

Our study showed that quercetin, beta-sitosterol, eugenol, palmitic acid, wogonin and Stigmasterol can match more targets, which were likely to be the key effective components of Xinyi San in treating rhinitis. Quercetin, as a flavonoid, is a research hotspot in recent years. It can significantly improve IL-2 and IFN- $\gamma$. Inhibit the levels of IL- 4 and IL-5, regulate the balance of Th1/Th2 cytokines, and have a protective effect on allergic rhinitis [6], and many experiments [7] [8] [9] have confirmed that quercetin has a significant effect on rhinitis. Beta sitosterol and Stigmasterol can reduce inflammatory cytokines IL-6 and TNF- $\alpha$ [10] [11]. Eugenol can reduce allergic reactions through suppression of cPLA2 and 5-L0 activation and through inhibition of COX-2 activity [12]. Toll-like receptors and their mediated innate immunity play an important role in the response of nasal sinus mucosal defense [13]. Palmitic acid has been shown to induce TLR4 


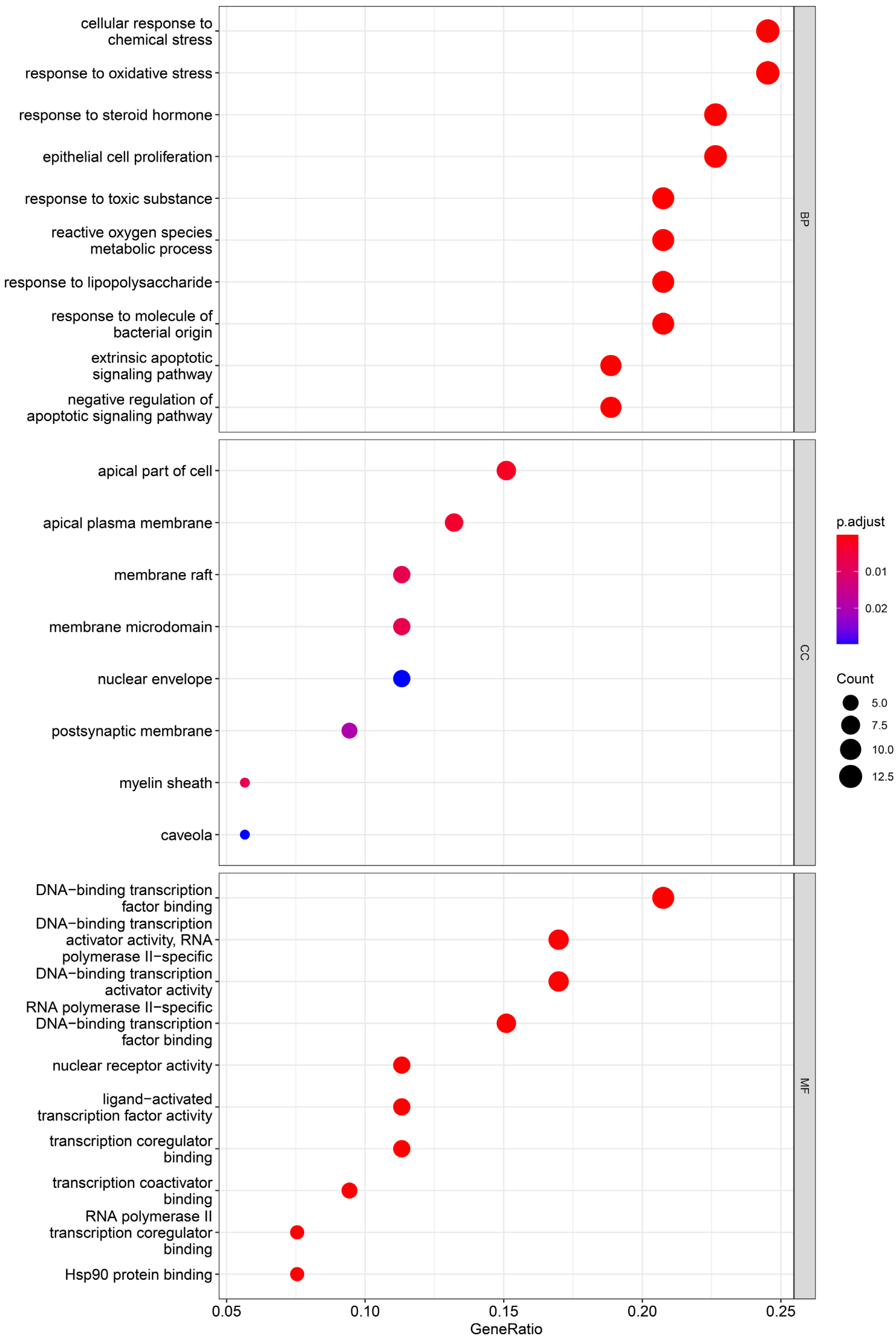

Figure 5. GO enrichment diagram of candidate targets of Xinyi San in the treatment of rhinitis. 


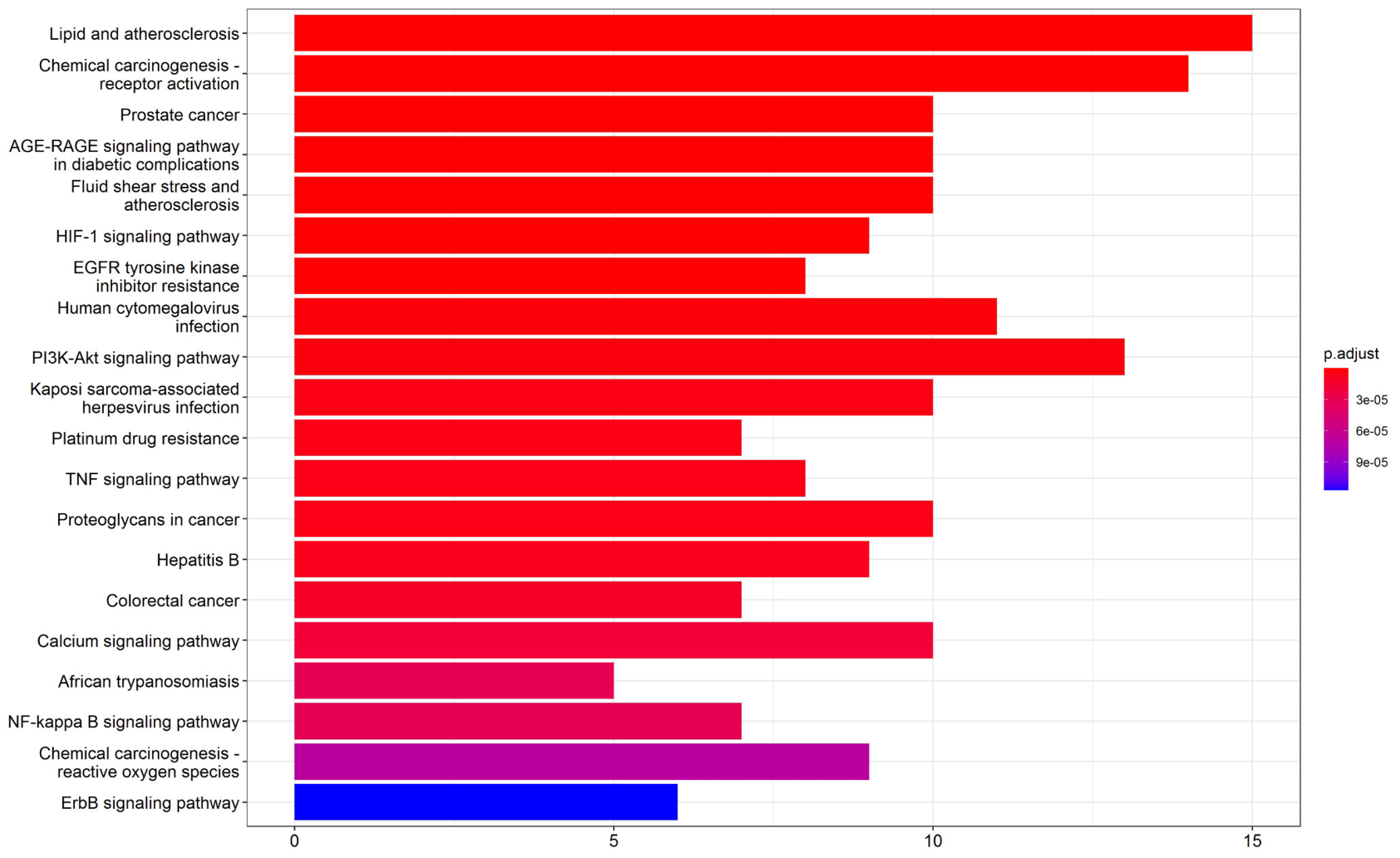

Figure 6. KEGG pathway enrichment analysis of potential target of Xinyi San-rhinitis

protein expression in vascular endothelial cells and significantly increase the TLR4 protein level of endothelial cell membrane, accompanied by significant activation of TLR4 inflammatory signaling pathway [14], which may have good therapeutic effect on allergic rhinitis; Wogonin was shown to be active chemicals in the anti Infammatory induced angiogenesis [15].

The PPI network shows that IL6, EGFR, VEGFA, HIF1A, CASP3, ESR1, PPARG, MYC, RELA and NFKBIA may be the key targets of Xinyi San in treating rhinitis. IL6 has been proved to be closely related to the occurrence and development of rhinitis [16] [17]. The activation of EGFR/EGF signaling pathway is inhibited, which can improve immune adhesion and affect the inflammatory response of AR nasal mucosa [18]. VEGF family mainly includes VEGFA, B, C, $\mathrm{D}, \mathrm{E}$ and $\mathrm{F}$, among which VEGFA is the most studied. Certain structural changes will occur in nasal mucosa of patients with rhinitis, and VEGF can promote angiogenesis, increase vascular permeability and vascular remodeling of nasal mucosa, promote damaged tissue repair and protect tissue cells [19]. HIF1A can regulate immunity, promote inflammation and induce neovascularization, and has a certain impact on the structural changes of nasal mucosa [20]. The experimental results of Li Yan et al. [21] suggest that the occurrence of rhinosinusitis may promote cell apoptosis by activating the apoptosis executive protein CASP3 through the upstream protein of the internal and external pathways of apoptosis. It is reported in the literature [22] that the prevalence of rhinitis in women is significantly higher than that in men, speculating that sex hormone ESR1 is of 
great significance in the occurrence and development of rhinitis. PPARG can regulate the inflammatory response of macrophages, mast cells, $\mathrm{T}$ cells and eosinophils, PPARG activator can reduce the rhinitis symptom score and inhibit the inflammatory response in allergic rhinitis mice [23]. MYC gene is a cell oncogene, which is involved in cell cycle regulation, cell growth regulation, cell proliferation, differentiation and transformation [24]. XIAO LF et al. [25] found that RELA, a gene known to be involved in regulating inflammation, can limit inflammatory response and treat rhinitis; NFKBIA plays a central role in inflammatory regulation and immune response [26], and has positive significance for the recovery of rhinitis.

GO enrichment analysis showed that the gene function of Xinyi San in the treatment of rhinitis was mainly reflected in DNA-binding transcription factor binding, (DNA-binding transcription activator activity, RNA polymerase II-specific), DNA-binding transcription activator activity, ubiquitin protein ligase binding, ubiquitin-like protein ligase binding, protein heterodimerization activity and so on. KEGG pathway enrichment analysis further confirmed the main signal pathways of Xinyi San in the treatment of rhinitis, mainly including Lipid and atherosclerosis, Chemical carcinogenesis - receptor activation and PI3K-Akt signaling pathway, which are mainly related to virus infection, inflammation and apoptosis, suggesting that Xinyi San may play on varieties of cytokines to play an anti-inflammatory and anti-inflammatory role Inhibit cytokine storm and treat rhinitis.

This study used network pharmacology to analyze the potential mechanism of Xinyi San in the treatment of rhinitis. The results confirmed the multi-component, multi-target and multi-channel characteristics of Xinyi San in the treatment of rhinitis, which provided a new idea for further experimental research and clinical application of Xinyi San.

\section{Conclusion}

In summary, we analyzed the main components of Xinyi San in treating rhinitis, and thus had a further understanding of its pharmacodynamic basis. By using the method of network pharmacology, the mechanism of Xinyi San in treating rhinitis was analyzed systematically at the molecular level. But the study also had some limitations, some of the results still needed to be verified by further laboratory tests and clinical studies.

\section{Funding}

This study was funded by Scientific Research Project of Hebei Administration of Traditional Chinese Medicine (No. 2022437).

\section{Conflicts of Interest}

The authors declare no conflicts of interest regarding the publication of this paper. 


\section{References}

[1] Hu, C., Li, H.W., Wu, L.J., et al. (2020) Mechanism of Cang'er San in Treatment of Rhinitis Based on Network Pharmacology. Chinese Archives of Traditional Chinese Medicine, 38, 64-71.

[2] Yan, Y.H. (1980) Redraft Yan's Jisheng Recipe. People's Health Publishing House, Beijing, 137.

[3] Wang, Y.H., Xu, Y., Cheng, Z.J., et al. (2008) Clinical Observation on 80 Cases of Children's Sinusitis Treated with Xinyi San. Zhejiang Journal of Traditional Chinese Medicine, 43, 42.

[4] Liu, L.J. (2017) Observation on 25 Cases of Rhinitis Treated with Modified Xinyi San and Xiaoqinglong Decoction. Nei Mongol Journal of Traditional Chinese Medicine, $1,24$.

[5] Xie, J., Gao, S., Li, L., et al. (2019) Research Progress and Application Strategy on Network Pharmacology in Chinese Materia Medica. Chinese Traditional and Herbal Drugs, 50, 2257-2265.

[6] Peng, W., Wang, W., Zhao, A.J., et al. (2018) Effects of Quercetin on Expressions of Th1/Th2 Cytokines in Serum of Experimental Allergic Rhinitis in Rats. Chinese Journal of Otorhinolaryngology-Skull Base Surgery, 24, 557-560.

[7] Sagit, M., Polat' H., Gurgen, S.G., Berk, E., Guler, S. and Yasar, M. (2017) Effectiveness of Quercetin in an Experimental Rat Model of Allergic Rhinitis. European Archives of Oto-Rhino-Laryngology, 274, 3087-3095. https://doi.org/10.1007/s00405-017-4602-Z

[8] Mlcek, J., Jurikova, T., Skrovankova, S., et al. (2016) Quercetin and Its Anti-Allergic Immune Response. Molecules, 21, Article No. 623.

https://doi.org/10.3390/molecules21050623

[9] Jafarinia, M., Hosseini, M.S., Kasiri, N., et al. (2020) Quercetin with the Potential Effect on Allergic Diseases. Allergy, Asthma \& Clinical Immunology, 16, Article No. 36. https://doi.org/10.1186/s13223-020-00434-0

[10] Choi, J.N., Choi, Y.H., Lee, J.M., et al. (2012) Anti-Inflammatory Effects of $\beta$-Sitosterol- $\beta$-D-Glucoside from Trachelospermum jasminoides (Apocynaceae) in Lipopolysaccharide-Stimulated RAW 264.7 Murine Macrophages. Natural Product Research, 26, 2340-2343. https://doi.org/10.1080/14786419.2012.654608

[11] Sabeva, N.S., McPhaul, C.M., Li, X.G., et al. (2011) Phytosterols Differentially Influence $\mathrm{ABC}$ Transporter Expression, Cholesterol Efflux and Inflammatory Cytokine Secretion in Macrophage Foam Cells. The Journal of Nutritional Biochemistry, 22, 777-783.

[12] Tang, F., Chen, F.L., Ling, X., et al. (2015) Inhibitory Effect of Methyleugenol on IgE-Mediated Allergic Inflammation in RBL-2H3 Cells. Mediators of Inflammation, 2015, Article ID: 463530. https://doi.org/10.1155/2015/463530

[13] Li, X.J. (2015) Toll-Like Receptors in the Research Progress of Rhinitis Nasal Polyps. Journal of Clinical and Pathological Research, 35, 1852-1856.

[14] Zhao, N.J., Wang, D.Q., Rong, Q.F., et al. (2013) Palmitate Enhances Toll-Like Receptor 4 Expression and Signaling in Porcine Vascular Endothelial Cells. Journal of Southern Medical University, 33, 1775-1777.

[15] Gong, G.W., Wang, H.Y., Kong, X.P., et al. (2018) Flavonoids Are Identified from the Extract of Scutellariae Radix to Suppress Inflammatory-Induced Angiogenic Responses in Cultured RAW 264.7 Macrophages. Scientific Reports, 8, Article No. 17412. https://doi.org/10.1038/s41598-018-35817-2

[16] Luo, J.H., Liao, F.G. and Liao, Z.P. (2010) Association the Polymorphism of Inter- 
leukin-6 in Patients with Allergic Rhinitis. Progress of Anatomical Sciences, 16, 442-444.

[17] Wang, B., Shu, Y., Liang, J., et al. (2016) Expression and Significance of Interleukin-6 in Children with Chronic Sinusitis. Chongqing Medicine, 45, 19-23.

[18] Han, R., Zheng, P.Y.' Wang, J.Q., et al. (2019) Desmoglein 3 Gene Mediates Epidermal Growth Factor/Epidermal Growth Factor Receptor Signaling Pathway Involved in Inflammatory Response and Immune Function of Anaphylactic Rhinitis. Biomedicine \& Pharmacotherapy, 118, Article ID: 109214.

[19] Guo, Q., Zou, S.Y., Xu, S.J., et al. (2020) The Histopathologic Changes and the Protein Expression of Vascular Endothelial Growth Factor in the Nasal Mucosa of Allergic Rhinitis. Journal of Harbin Medical University, 54, 546-548.

[20] Baek, K.J., Cho, J.Y., Rosenthal, P., et al. (2013) Hypoxia Potentiates Allergen Induction of HIF- $1 \alpha$, Chemokines, Airway Inflammation, TGF- $\beta 1$, and Airway Remodeling in a Mouse Model. Clinical Immunology, 147, 27-37.

[21] Li, Y., Zhang, J.F. and Wang, D.Y. (2018) Effects of Qingbi Pills on the Expression of IL- 8 mRNA and TNF- $\alpha$ mRNA of Nasal Mucosa Tissue in the Rabbits of Rhinosinusitis. World Journal of Integrated Traditional and Western Medicine, 13, 1095-1098.

[22] Klis, K. and Wronka, I. (2017) Association of Estrogen-Related Traits with Allergic Rhinitis. In: Pokorski, M., Ed., Influenza and Respiratory Care, Springer, Cham, 71-78. https://doi.org/10.1007/5584 2016190

[23] Lee, J.E., Zhang, Y.L., Han, D.H., et al. (2015) Antiallergic Function of KR62980, a Peroxisome Proliferator-Activated Receptor- $\gamma$ Agonist, in a Mouse Allergic Rhinitis Model. Allergy, Asthma \& Immunology Research, 7, 256-264.

[24] Guo, X.J., Cheng, Z.Q. and Jin, H.T. (2017) Expression of c-myc and Ki-67 and Their Significance in Extranodal NK/T-Cell Lymphoma, Nasal Type. Chinese Journal of Diagnostic Pathology, 24, 517-520.

[25] Xiao, L.F., Jiang, L., Hu, Q., et al. (2018) MiR-302e Attenuates Allergic Inflammation Model by Targeting RelA. Bioscience Reports, 38, Article ID: BSR20180025. https://doi.org/10.1042/BSR20180025

[26] Guan, H.J., Sun, G.F. and Xie, H.F. (2019) Genetic Loci in the NF- $\kappa B$ Pathway and Lung Cancer Susceptibility: A Meta-Analysis. Chinese Journal of Evidence-Based Medicine, 19, 1430-1435. 